PROCEEDINGS OF THE

AMERICAN MATHEMATICAL SOCIETY

Volume 136, Number 11, November 2008, Pages 3983-3991

S 0002-9939(08)09394-5

Article electronically published on June 11, 2008

\title{
INCLUSION THEOREMS FOR ABSOLUTELY SUMMING HOLOMORPHIC MAPPINGS
}

\author{
HEINZ JUNEK, MÁRIO C. MATOS, AND DANIEL PELLEGRINO
}

(Communicated by N. Tomczak-Jaegermann)

\begin{abstract}
For linear operators, if $1 \leq p \leq q<\infty$, then every absolutely $p$-summing operator is also absolutely $q$-summing. On the other hand, it is well known that for $n \geq 2$, there are no general "inclusion theorems" for absolutely summing $n$-linear mappings or $n$-homogeneous polynomials. In this paper we deal with situations in which the spaces of absolutely $p$-summing and absolutely $q$-summing linear operators coincide, and prove that for $1 \leq$ $p \leq q \leq 2$ and $n \geq 2$, we have inclusion theorems for absolutely summing $n$-linear mappings $/ n$-homogeneous polynomials/holomorphic mappings. It is worth mentioning that our results hold precisely in the opposite direction from what is expected in the linear case, i.e., we show that, in some situations, as $p$ increases, the classes of absolutely $p$-summing mappings becomes smaller.
\end{abstract}

\section{INTRODUCTION}

The theory of absolutely $p$-summing operators goes back to 1950, with Grothendieck's seminal paper [7. In the sixties, with the works of Pietsch [14] and Lindenstrauss-Pełczyński [8], absolutely $p$-summing linear operators have become an important field of investigation in functional analysis. For the general theory of absolutely $p$-summing linear operators, we refer to the book by DiestelJarchow-Tonge 6. From now on, the ideal of absolutely $p$-summing linear operators will be denoted by $\mathcal{L}_{a s, p}$, and if $T: X \rightarrow Y$ is absolutely $p$-summing, we write $T \in \mathcal{L}_{a s, p}(X ; Y)$.

It is well known that, if $1 \leq p \leq q$, then every absolutely $p$-summing linear operator is absolutely $q$-summing. Results of this type are called "inclusion theorems".

Since Pietsch's paper [15], several generalizations of absolutely summing operators to polynomials and multilinear mappings were introduced, such as absolutely summing polynomials and $n$-linear mappings ([1, 9]) and fully (or multiple) summing $n$-linear mappings (3, 11]). We will recall the necessary definitions in Sections 2 and 3.

For fully summing $n$-linear mappings, there is an inclusion theorem for some particular situation (see 13]), asserting that if an $n$-linear mapping is fully $p$ summing and $1 \leq p \leq q<2$, then it is fully $q$-summing. But, for absolutely summing mappings there is no inclusion theorem. Indeed, for $n \geq 2$, we have

Received by the editors December 29, 2006, and, in revised form, October 18, 2007.

2000 Mathematics Subject Classification. Primary 46B15; Secondary 46G25.

The third author was supported by CNPq Grants 471054/2006-2 (Edital Universal) and 308084/2006-3.

(C)2008 American Mathematical Society Reverts to public domain 28 years from publication 
$\mathcal{L}\left({ }^{n} l_{2}, \mathbb{C}\right)=\mathcal{L}_{a s, 1}\left({ }^{n} l_{2}, \mathbb{C}\right)$ but $\mathcal{L}\left({ }^{n} l_{2}, \mathbb{C}\right) \neq \mathcal{L}_{a s, 2}\left({ }^{n} l_{2}, \mathbb{C}\right)$, and thus $\mathcal{L}_{a s, 1}\left({ }^{n} l_{2}, \mathbb{C}\right)$ is not contained in $\mathcal{L}_{a s, 2}\left({ }^{n} l_{2}, \mathbb{C}\right)$. In the linear case, the situation is sometimes simpler and we have more than just inclusions: if $X$ has cotype 2 and $1 \leq r \leq q \leq 2$, then

$$
\mathcal{L}_{a s, q}(X ; Y)=\mathcal{L}_{a s, r}(X ; Y)
$$

for every $Y$. Surprisingly, we will show that (if $X$ has cotype 2 and $n \geq 2$ ) there are inclusion theorems, but they occur in the opposite direction from what is usual in the linear case; i.e., we will show that if $p$ increases, the ideal decreases. For example, we prove that if $X$ has cotype 2 and $n \geq 2$, then

$$
\mathcal{L}_{a s, q}\left({ }^{n} X ; Y\right) \subseteq \mathcal{L}_{a s, r}\left({ }^{n} X ; Y\right),
$$

for every $Y$ and $1 \leq r \leq q \leq 2$. We also obtain similar results for homogeneous polynomials and holomorphic mappings.

In the following, $X, X_{1}, \ldots, X_{n}, Y$ will denote Banach spaces over the complex scalar field and $\mathbb{N}$ will denote the set of all positive integers. By $X^{\prime}$ we denote the topological dual of $X$, and $B_{X^{\prime}}$ represents its closed unit ball.

For $p \geq 1$, the vector space of all sequences $\left(x_{j}\right)_{j=1}^{\infty}$ in $X$ such that $\left\|\left(x_{j}\right)_{j=1}^{\infty}\right\|_{p}=$ $\left(\sum_{j=1}^{\infty}\left\|x_{j}\right\|^{p}\right)^{\frac{1}{p}}<\infty$ is denoted by $l_{p}(X)$. We denote by $l_{p}^{w}(X)$ the linear space of the sequences $\left(x_{j}\right)_{j=1}^{\infty}$ in $X$ such that $\left(\varphi\left(x_{j}\right)\right)_{j=1}^{\infty} \in l_{p}$ for every continuous linear functional $\varphi: X \rightarrow \mathbb{C}$. This is a Banach space under the norm

$$
\left\|\left(x_{j}\right)_{j=1}^{\infty}\right\|_{w, p}=\sup _{\varphi \in B_{X^{\prime}}}\left\|\left(\varphi\left(x_{j}\right)\right)_{j=1}^{\infty}\right\|_{p} .
$$

As usual, $l_{p}^{u}(X)$ denotes the subspace of $l_{p}^{w}(X)$ composed by the sequences $\left(x_{j}\right)_{j=1}^{\infty} \in$ $l_{p}^{w}(X)$ such that

$$
\lim _{k \rightarrow \infty}\left\|\left(x_{j}\right)_{j=k}^{\infty}\right\|_{w, p}=0 .
$$

Given a natural number $n \in \mathbb{N}$, the Banach space of all continuous $n$-linear mappings from $X$ to $Y$ endowed with the sup norm will be denoted by $\mathcal{L}\left({ }^{n} X ; Y\right)$ $(\mathcal{L}(X ; Y)$ if $n=1)$. The set of all continuous $n$-homogeneous polynomials with the sup norm will be denoted by $\mathcal{P}\left({ }^{n} X ; Y\right)$.

The paper is organized as follows: In Section 2 we obtain connections between the theory of absolutely summing holomorphic mappings and absolutely summing polynomials; in Section 3, by using multilinear interpolation techniques, we obtain inclusion theorems for absolutely summing multilinear mappings; in Section 4 we adapt our results to homogeneous polynomials; and, in the last section, the results are extended to holomorphic mappings.

\section{Absolutely Summing holomorphic MAPPings}

The theory of absolutely summing holomorphic mappings was introduced by the second author in [10].

From now on, $A$ will always be an open set of $X$ and $p, q, r, s$ will be real numbers greater than or equal to 1 . If $a \in A, B_{\delta}(a)$ will denote the closed ball of center $a$ and radius $\delta$.

Definition 1. A holomorphic mapping $f: A \subset X \rightarrow Y$ at a point $a \in A$ is absolutely $(r ; s)$-summing at $a$ if there is a $\delta>0$ such that $B_{\delta}(a) \subset A$ and

$$
\left(f\left(a+x_{j}\right)-f(a)\right)_{j=1}^{\infty} \in l_{r}(Y)
$$

whenever $\left(x_{j}\right)_{j=1}^{\infty} \in l_{s}^{u}(X)$ with $\left\|x_{j}\right\| \leq \delta$ for every $j \in \mathbb{N}$. 
If $f$ is an $n$-homogeneous polynomial and $a=0$, it is equivalent to saying that $\left(f\left(x_{j}\right)\right)_{j=1}^{\infty} \in l_{r}(Y)$ whenever $\left(x_{j}\right)_{j=1}^{\infty} \in l_{s}^{u}(X)$. The following characterization will be useful:

Theorem 1 ([9]). If $P \in \mathcal{P}\left({ }^{n} X ; Y\right)$, the following statements are equivalent:

(1) $P$ is absolutely $(r ; s)$-summing.

(2) There exists $L>0$ such that

$$
\left(\sum_{j=1}^{\infty}\left\|P\left(x_{j}\right)\right\|^{r}\right)^{\frac{1}{r}} \leq L\left\|\left(x_{j}\right)_{j=1}^{\infty}\right\|_{w, s}^{n} \text { for all }\left(x_{j}\right)_{j=1}^{\infty} \in l_{s}^{u}(X) .
$$

The infimum of the possible constants $L>0$ (denoted by $\left.\|\cdot\|_{a s(r ; s)}\right)$ is a norm for the space of absolutely $(r ; s)$-summing polynomials (represented as $\mathcal{P}_{a s(r ; s)}\left({ }^{n} X ; Y\right)$ ).

For the theory of absolutely summing polynomials we refer to [9].

If $a \in X$, let us denote by $\mathcal{H}_{a s(r ; s)}^{(a)}(X ; Y)$ the set of all holomorphic mappings at $a \in X$ which are absolutely $(r ; s)$-summing at $a$. The next theorem (that will be very useful in the final section) states a nice connection between the study of absolutely summing holomorphic mappings and absolutely summing homogeneous polynomials.

Theorem 2. A holomorphic mapping $f: A \subset X \rightarrow Y$ at a point $a \in A$ belongs to $\mathcal{H}_{a s(r ; s)}^{(a)}(X ; Y)$ if, and only if,

$$
\frac{1}{n !} \widehat{d}^{n} f(a) \in \mathcal{P}_{a s(r ; s)}\left({ }^{n} X ; Y\right)
$$

and there are finite constants $K \geq 0$ and $k>0$ such that

$$
\left\|\frac{1}{n !} \widehat{d}^{n} f(a)\right\|_{a s(r ; s)} \leq K k^{n}
$$

for every $n \in \mathbb{N}$.

Proof. Let us suppose that (2.1) and (2.2) hold. Since $f$ is holomorphic at $a$, there is $\delta>0$ such that

$$
f(a+x)-f(a)=\sum_{n=1}^{\infty} \frac{1}{n !} \widehat{d}^{n} f(a)(x)
$$

for every $x \in E$ with $\|x\| \leq \delta$.

If $\left(x_{j}\right)_{j=1}^{\infty} \in l_{s}^{u}(X)$, with

$$
\left\|\left(x_{j}\right)_{j=1}^{\infty}\right\|_{w, s}<\min \left\{\delta, \frac{1}{2 k}\right\}
$$

we have

$$
\begin{aligned}
\left\|\left(f\left(a+x_{j}\right)-f(a)\right)_{j=1}^{\infty}\right\|_{r} & \leq \sum_{n=1}^{\infty}\left\|\left(\frac{1}{n !} \widehat{d}^{n} f(a)\left(x_{j}\right)\right)_{j=1}^{\infty}\right\|_{r} \leq K \sum_{n=1}^{\infty}\left(k\left\|\left(x_{j}\right)_{j=1}^{\infty}\right\|_{w, s}\right)^{n} \\
& \leq K \sum_{n=1}^{\infty} k^{n} \frac{1}{(2 k)^{n}}<\infty
\end{aligned}
$$

and hence $f$ is absolutely summing at $a$. 
Conversely, suppose that $f$ is absolutely summing at $a$. Hence, from [10, Theorem 3.5], there are $C_{1} \geq 0$ and $\delta_{1}>0$ such that

$$
f(a+x)-f(a)=\sum_{n=1}^{\infty} \frac{1}{n !} \widehat{d}^{n} f(a)(x)
$$

for $x \in E$ with $\|x\| \leq \delta_{1}$, and

$$
\left\|\left(f\left(a+z_{j}\right)-f(a)\right)_{j=1}^{\infty}\right\|_{r}^{r} \leq C_{1}\left\|\left(z_{j}\right)_{j=1}^{\infty}\right\|_{w, s}^{s}
$$

for $\left(z_{j}\right)_{j=1}^{\infty} \in l_{s}^{u}(X)$ with $\left\|\left(z_{j}\right)_{j=1}^{\infty}\right\|_{w, s} \leq \delta_{1}$.

So, if $\left(z_{j}\right)_{j=1}^{\infty} \in l_{s}^{u}(X)$ and $0 \neq\left\|\left(z_{j}\right)_{j=1}^{\infty}\right\|_{w, s} \leq \delta_{1}$, we have, for every $n$,

$$
\begin{aligned}
\sum_{j=1}^{\infty}\left\|\frac{1}{n !} \widehat{d}^{n} f(a)\left(z_{j}\right)\right\|^{r} & =\sum_{j=1}^{\infty}\left\|\frac{1}{2 \pi i} \int_{|\lambda|=1} \frac{f\left(a+\lambda z_{j}\right)-f(a)}{\lambda^{n+1}} d \lambda\right\|^{r} \\
& \leq \sum_{j=1}^{\infty} \sup _{|\lambda|=1}\left\|f\left(a+\lambda z_{j}\right)-f(a)\right\|^{r} \\
& \leq C_{1} \sup _{|\lambda|=1}\left\|\left(\lambda z_{j}\right)_{j=1}^{\infty}\right\|_{w, s}^{s}=C_{1}\left\|\left(z_{j}\right)_{j=1}^{\infty}\right\|_{w, s}^{s} .
\end{aligned}
$$

Hence $\frac{1}{n !} \widehat{d}^{n} f(a) \in \mathcal{P}_{a s(r ; s)}\left({ }^{n} X ; Y\right)$.

To simplify notation, we will write $P_{n}=\frac{1}{n !} \widehat{d}^{n} f(a)$.

If $0 \neq\left(x_{j}\right)_{j=1}^{\infty} \in l_{s}^{u}(X)$, we have

$$
\left\|\left(\frac{\delta_{1} x_{j}}{\left\|\left(x_{t}\right)_{t=1}^{\infty}\right\|_{w, s}}\right)_{j=1}^{\infty}\right\|_{w, s}=\delta_{1}
$$

and thus

$$
\sum_{j=1}^{\infty}\left\|P_{n}\left(\frac{\delta_{1} x_{j}}{\left\|\left(x_{t}\right)_{t=1}^{\infty}\right\|_{w, s}}\right)\right\|^{r} \leq C_{1}\left\|\left(\frac{\delta_{1} x_{j}}{\left\|\left(x_{t}\right)_{t=1}^{\infty}\right\|_{w, s}}\right)_{j=1}^{\infty}\right\|_{w, s}^{s}
$$

Hence

$$
\left(\sum_{j=1}^{\infty}\left\|P_{n}\left(\frac{\delta_{1} x_{j}}{\left\|\left(x_{t}\right)_{t=1}^{\infty}\right\|_{w, s}}\right)\right\|^{r}\right)^{\frac{1}{r}} \leq C_{1}^{\frac{1}{r}}\left\|\left(\frac{\delta_{1} x_{j}}{\left\|\left(x_{t}\right)_{t=1}^{\infty}\right\|_{w, s}}\right)_{j=1}^{\infty}\right\|_{w, s}^{\frac{s}{r}},
$$

and we obtain

$$
\left(\sum_{j=1}^{\infty}\left\|P_{n}\left(x_{j}\right)\right\|^{r}\right)^{\frac{1}{r}} \leq C_{1}^{\frac{1}{r}} \frac{\left\|\left(x_{t}\right)_{t=1}^{\infty}\right\|_{w, s}^{n}}{\delta_{1}^{n}} \delta_{1}^{s / r}=\left(C_{1}^{\frac{1}{r}} \delta_{1}^{s / r}\right)\left(\frac{1}{\delta_{1}}\right)^{n}\left\|\left(x_{t}\right)_{t=1}^{\infty}\right\|_{w, s}^{n}
$$

and finally

$$
\left\|\frac{1}{n !} \widehat{d}^{n} f(a)\right\|_{a s(r, s)} \leq\left(C_{1}^{\frac{1}{r}} \delta_{1}^{s / r}\right)\left(\frac{1}{\delta_{1}}\right)^{n}
$$

for every $n \in \mathbb{N}$. 


\section{INCLUSION THEOREM FOR MULTILINEAR MAPPINGS}

Recall that an $n$-linear map $T \in \mathcal{L}\left({ }^{n} X ; Y\right)$ is absolutely $(r ; s)$-summing $(T \in$ $\left.\mathcal{L}_{a s(r ; s)}\left({ }^{n} X ; Y\right)\right)$ if

$$
T\left(x_{j}^{(1)}, \ldots, x_{j}^{(n)}\right)_{j=1}^{\infty} \in l_{r}(Y)
$$

whenever $\left(x_{j}^{(k)}\right)_{j=1}^{\infty} \in l_{s}^{w}(X), k=1, \ldots, n$. In the case $r=s$ we simply say that $T$ is $r$-summing, and instead of $\mathcal{L}_{a s(r ; r)}$ we write $\mathcal{L}_{a s, r}$. The characterization of absolutely $r$-summing mappings and the definition of the norm is similar to those of Theorem 1 (for details we refer to [1]). In particular, the following characterization of absolutely $r$-summing mappings holds true:

Proposition 1. $T \in \mathcal{L}\left({ }^{n} X ; Y\right)$ is absolutely r-summing if and only if the mapping

$$
\begin{aligned}
\widehat{T}: l_{r}^{u}(X) \times \cdots \times l_{r}^{u}(X) \rightarrow & l_{r}(Y) \text { given by } \\
& \widehat{T}\left(\left(x_{j}^{(1)}\right)_{j=1}^{\infty}, \ldots,\left(x_{j}^{(n)}\right)_{j=1}^{\infty}\right)=\left(T\left(x_{j}^{(1)}, \ldots, x_{j}^{(n)}\right)\right)_{j=1}^{\infty}
\end{aligned}
$$

is well defined and bounded. In this case we have

$$
\|T\|_{a s, r}=\|\widehat{T}\| \text {. }
$$

Now, we are ready to prove an inclusion theorem. As usual, the number $C_{2}(X)$ denotes the cotype constant of the Banach space $X$.

Theorem 3 (Inclusion Theorem). If $X$ has cotype 2 and $n \geq 2$, then

$$
\mathcal{L}_{a s, q}\left({ }^{n} X ; Y\right) \subseteq \mathcal{L}_{a s, r}\left({ }^{n} X ; Y\right)
$$

holds true for $1 \leq r \leq q \leq 2$. Besides,

$$
\|\cdot\|_{a s, r} \leq 16^{n} C_{2}(X)^{\left(\frac{7}{2}-\theta\right) n}\|\cdot\|^{1-\theta}\|\cdot\|_{a s, q}^{\theta},
$$

where $\theta$ is given by

$$
\frac{1}{r}=(1-\theta)+\frac{\theta}{q}
$$

Proof. From [4, Theorem 2.5] we know that

$$
\mathcal{L}_{a s, 1}\left({ }^{n} X ; Y\right)=\mathcal{L}\left({ }^{n} X ; Y\right) \text { and }\|\cdot\|_{a s, 1} \leq C_{2}(X)^{n}\|\cdot\|
$$

hold true for $n \geq 2$ and all Banach spaces $Y$, provided that $X$ has cotype 2. So it suffices to prove the following sandwich-type result and then to apply it to $p=1$ :

- If $X$ has cotype 2 and $T \in \mathcal{L}_{a s, p}\left({ }^{n} X ; Y\right) \cap \mathcal{L}_{a s, q}\left({ }^{n} X ; Y\right)$ for some $1 \leq p \leq$ $q \leq 2$, then

$$
T \in \mathcal{L}_{a s, r}\left({ }^{n} X ; Y\right)
$$

for every $r$ with $p \leq r \leq q$. Besides,

$$
\|\cdot\|_{a s, r} \leq 16^{n} C_{2}(X)^{5 n / 2}\|\cdot\|_{a s, p}^{1-\theta}\|\cdot\|_{a s, q}^{\theta},
$$

with $\theta$ given by

$$
\frac{1}{r}=\frac{1-\theta}{p}+\frac{\theta}{q}
$$


We are going to prove the above result using the interpolation technique. By assumption on $T$ and by Proposition 1 the map $T$ generates bounded operators

$$
\left\{\begin{array}{l}
\widehat{T_{p}}: l_{p}^{u}(X) \times \cdots \times l_{p}^{u}(X) \rightarrow l_{p}(Y), \\
\widehat{T_{q}}: l_{q}^{u}(X) \times \cdots \times l_{q}^{u}(X) \rightarrow l_{q}(Y) .
\end{array}\right.
$$

Applying the complex interpolation method to these $n$-linear operators we get a linear operator

$$
\widehat{T_{(\theta)}}:\left[l_{p}^{u}(X), l_{q}^{u}(X)\right]_{\theta} \times \cdots \times\left[l_{p}^{u}(X), l_{q}^{u}(X)\right]_{\theta} \rightarrow\left[l_{p}(Y), l_{q}(Y)\right]_{\theta}
$$

with

$$
\left\|\widehat{T_{(\theta)}}\right\| \leq\left\|\widehat{T_{p}}\right\|^{1-\theta}\left\|\widehat{T_{q}}\right\|^{\theta}
$$

This operator satisfies

$$
\widehat{T_{(\theta)}}\left(\left(x_{j}^{(1)}\right)_{j=1}^{\infty}, \ldots,\left(x_{j}^{(n)}\right)_{j=1}^{\infty}\right)=\left(T\left(x_{j}^{(1)}, \ldots, x_{j}^{(n)}\right)\right)_{j=1}^{\infty}
$$

for all sequences $\left(x_{j}^{(k)}\right)_{j=1}^{\infty}$ in $\left[l_{p}^{u}(X), l_{q}^{u}(X)\right]_{\theta}, 1 \leq k \leq n$.

By [2] we have $\left[l_{p}(Y), l_{q}(Y)\right]_{\theta}=l_{r}(Y)$ isometrically (with $r$ as in (3.1)), and, using the natural isometric identification $l_{p}^{u}(X)=l_{p} \widetilde{\otimes}_{\varepsilon} X$, we obtain from [5, Lemma 2 and Proposition 8], as a particular case, a natural isomorphism

$$
J: l_{r}^{u}(X) \rightarrow\left[l_{p}^{u}(X), l_{q}^{u}(X)\right]_{\theta}
$$

with

$$
\|J\| \leq 16 C_{2}(X)^{\frac{5}{2}} .
$$

With this isomorphism we can identify the operator $\widehat{T_{(\theta)}}$ with the map

$$
\widehat{T_{r}}: l_{r}^{u}(X) \times \cdots \times l_{r}^{u}(X) \rightarrow l_{r}(Y),
$$

and this gives us $T \in \mathcal{L}_{a s, r}\left({ }^{n} X ; Y\right)$ and

$$
\begin{aligned}
\|T\|_{a s, r} & =\left\|\widehat{T_{r}}\right\| \leq\|J\|^{n}\left\|\widehat{T_{(\theta)}}\right\| \leq 16^{n} C_{2}(X)^{\frac{5 n}{2}}\left\|\widehat{T_{p}}\right\|^{1-\theta}\left\|\widehat{T_{q}}\right\|^{\theta} \\
& =16^{n} C_{2}(X)^{\frac{5 n}{2}}\|T\|_{a s, p}^{1-\theta}\|T\|_{a s, q}^{\theta} .
\end{aligned}
$$

In our special case, $p=1$ and $\|T\|_{a s, 1} \leq C_{2}(X)^{n}\|T\|$. Hence

$$
\|T\|_{a s, r} \leq 16^{n} C_{2}(X)^{\frac{5 n}{2}}\left(C_{2}(X)^{n}\|T\|\right)^{1-\theta}\|T\|_{a s, q}^{\theta},
$$

and the result follows.

Remark 1. In the linear case, for spaces $X$ of cotype 2 we have $\mathcal{L}_{a s, r}(X ; Y)=$ $\mathcal{L}_{a s, 2}(X ; Y)$ for all numbers $r$ with $1 \leq r \leq 2$ (cf. [6, Corollary 11.16]).

Remark 2. The same reasoning can be adapted to $n$-linear mappings defined on $X_{1} \times \cdots \times X_{n}$ instead of $X \times \cdots \times X$. 


\section{INCLUSION THEOREM FOR POLYNOMIALS}

If $P \in \mathcal{P}\left({ }^{n} X ; Y\right)$, we denote by $\stackrel{\vee}{P}$ the symmetric $n$-linear mapping associated to $P$. The following simple result will be useful in this section.

Proposition 2. Let $n \in \mathbb{N}$ and $s \geq 1$. If $P \in \mathcal{P}_{a s, s}\left({ }^{n} X ; Y\right)$, then $\stackrel{\vee}{P} \in \mathcal{L}_{a s, s}\left({ }^{n} X ; Y\right)$ and $\|\stackrel{\vee}{P}\|_{a s, s} \leq e^{n}\|P\|_{a s, s}$.

Proof. If $\left(x_{j}^{(1)}\right)_{j=1}^{\infty}, \ldots,\left(x_{j}^{(n)}\right)_{j=1}^{\infty} \in B_{l_{s}^{u}(X)}$, then, by invoking the Polarization Formula, for every $j$, we obtain

$$
\stackrel{\vee}{P}\left(x_{j}^{(1)}, \ldots, x_{j}^{(n)}\right)=\frac{1}{2^{n} n !} \sum_{\varepsilon_{i}=-1,1} \varepsilon_{1} \cdots \varepsilon_{n} P\left(\varepsilon_{1} x_{j}^{(1)}+\cdots+\varepsilon_{n} x_{j}^{(n)}\right) .
$$

So, we can infer that

$$
\begin{aligned}
& \left(\sum_{j=1}^{\infty}\left\|\stackrel{\vee}{P}\left(x_{j}^{(1)}, \ldots, x_{j}^{(n)}\right)\right\|^{s}\right)^{\frac{1}{s}} \\
& =\frac{1}{2^{n} n !}\left(\sum_{j=1}^{\infty}\left\|\sum_{\varepsilon_{i}=-1,1} \varepsilon_{1} \cdots \varepsilon_{n} P\left(\varepsilon_{1} x_{j}^{(1)}+\cdots+\varepsilon_{n} x_{j}^{(n)}\right)\right\|^{s}\right)^{\frac{1}{s}} \\
& \leq \frac{1}{2^{n} n !} \sum_{\varepsilon_{i}=-1,1}\left(\sum_{j=1}^{\infty}\left\|P\left(\varepsilon_{1} x_{j}^{(1)}+\cdots+\varepsilon_{n} x_{j}^{(n)}\right)\right\|^{s}\right)^{\frac{1}{s}} \\
& \leq \frac{1}{2^{n} n !}\|P\|_{a s, s} \sum_{\varepsilon_{i}=-1,1}\left\|\left(\varepsilon_{1} x_{j}^{(1)}+\cdots+\varepsilon_{n} x_{j}^{(n)}\right)_{j=1}^{\infty}\right\|_{w, s}^{n} \\
& \leq \frac{1}{2^{n} n !}\|P\|_{a s, s} 2^{n}\left(\left\|\left(x_{j}^{(1)}\right)_{j=1}^{\infty}\right\|_{w, s}+\cdots+\left\|\left(x_{j}^{(n)}\right)_{j=1}^{\infty}\right\|_{w, s}\right)^{n} \\
& \leq \frac{1}{n !}\|P\|_{a s, s} n^{n} \leq e^{n}\|P\|_{a s, s} \cdot
\end{aligned}
$$

Theorem 4 (Inclusion Theorem). If $X$ has cotype 2 and $n \geq 2$, then

$$
\mathcal{P}_{a s, q}\left({ }^{n} X ; Y\right) \subseteq \mathcal{P}_{a s, r}\left({ }^{n} X ; Y\right)
$$

holds true for $1 \leq r \leq q \leq 2$ and all Banach spaces $Y$. Besides,

$$
\|\cdot\|_{a s, r} \leq(16 e)^{n} C_{2}(X)^{\left(\frac{7}{2}-\theta\right) n}\|\cdot\|^{1-\theta}\|\cdot\|_{a s, q}^{\theta},
$$

where $\theta$ is given by

$$
\frac{1}{r}=(1-\theta)+\frac{\theta}{q}
$$

Proof. Let $P \in \mathcal{P}_{a s, q}\left({ }^{n} X ; Y\right)$. From Proposition 2 we know that $\stackrel{\vee}{P} \in \mathcal{L}_{a s, q}\left({ }^{n} X ; Y\right)$, and from Theorem 3 we deduce that

$$
\stackrel{\vee}{P} \in \mathcal{L}_{a s, r}\left({ }^{n} X ; Y\right)
$$


and

$$
\|\stackrel{\vee}{P}\|_{a s, r} \leq 16^{n} C_{2}(X)^{\left(\frac{7}{2}-\theta\right) n}\|\stackrel{\vee}{P}\|^{1-\theta}\|\stackrel{\vee}{P}\|_{a s, q}^{\theta}
$$

with

$$
\frac{1}{r}=(1-\theta)+\frac{\theta}{q}
$$

So,

$$
\begin{aligned}
\|P\|_{a s, r} & \leq\|\stackrel{\vee}{P}\|_{a s, r} \leq 16^{n} C_{2}(X)^{\left(\frac{7}{2}-\theta\right) n}\|\stackrel{\vee}{P}\|^{1-\theta}\|\stackrel{\vee}{P}\|_{a s, q}^{\theta} \\
& \leq 16^{n} C_{2}(X)^{\left(\frac{7}{2}-\theta\right) n}\left(e^{n}\|P\|\right)^{1-\theta}\left(e^{n}\|P\|_{a s, q}\right)^{\theta} \\
& =(16 e)^{n} C_{2}(X)^{\left(\frac{7}{2}-\theta\right) n}\|P\|^{1-\theta}\|P\|_{a s, q}^{\theta} .
\end{aligned}
$$

\section{INCLUSION THEOREM FOR HOLOMORPHIC MAPPINGS}

The next theorem extends the previous results for holomorphic mappings.

Theorem 5. If $1 \leq r \leq q \leq 2$ and $X$ has cotype 2 , then

$$
\mathcal{H}_{a s, q}^{(a)}(X ; Y) \subseteq \mathcal{H}_{a s, r}^{(a)}(X ; Y)
$$

for all Banach spaces $Y$.

Proof. If $f \in \mathcal{H}_{a s, q}^{(a)}(X ; Y)$, from Theorem 2 we know that there are positive constants $k_{1}$ and $K_{1}$ such that

$$
\left\|\frac{1}{m !} \widehat{d}^{m} f(a)\right\|_{a s, q} \leq K_{1} k_{1}^{m}
$$

for every $m \in \mathbb{N}$. Hence, using Theorem 4 we get, for $m \geq 2$,

$$
\left\|\frac{1}{m !} \widehat{d}^{m} f(a)\right\|_{a s, r} \leq(16 e)^{m} C_{2}(X)^{\left(\frac{7}{2}-\theta\right) m}\left\|\frac{1}{m !} \widehat{d}^{m} f(a)\right\|^{1-\theta}\left\|\frac{1}{m !} \widehat{d}^{m} f(a)\right\|_{a s, q}^{\theta} \leq K_{2} k_{2}^{m} .
$$

From Remark 1, we know that

$$
d f(a) \in \mathcal{L}_{a s, q}(X ; Y)=\mathcal{L}_{a s, r}(X ; Y),
$$

and we can easily find adequate constants $k_{3}$ and $K_{3}$ such that

$$
\left\|\frac{1}{m !} \widehat{d}^{m} f(a)\right\|_{a s, r} \leq K_{3} k_{3}^{m}
$$

for every $m \in \mathbb{N}$. Hence Theorem 2 asserts that $f \in \mathcal{H}_{a s, r}^{(a)}(X ; Y)$.

\section{ACKNOWLedGement}

The authors thank the referee for a careful examination of the paper and helpful comments. 


\section{REFERENCES}

[1] R. Alencar and M. C. Matos, Some classes of multilinear mappings between Banach spaces, Publicaciones Universidad Complutense de Madrid 12 (1989).

[2] J. Bergh and J. Löfström, Interpolation spaces, Springer-Verlag, 1976. MR0482275 (58:2349)

[3] F. Bombal, D. Pérez-García and I. Villanueva, Multilinear extensions of Grothendieck's theorem, Quart. J. Math. 55 (2004), 441-450. MR2104683 (2005i:47032)

[4] G. Botelho, Cotype and absolutely summing multilinear mappings and homogeneous polynomials, Proc. Royal Irish Acad. 97 (1997), 145-153. MR1645283 (99i:46006)

[5] A. Defant and C. Michels, A complex interpolation formula for tensor products of vectorvalued Banach function spaces, Arch. Math. 74 (2000), 441-451. MR1753543(2001d:46103)

[6] J. Diestel, H. Jarchow and A. Tonge, Absolutely summing operators, Cambridge University Press, 1995. MR 1342297 (96i:46001)

[7] A. Grothendieck, Resumé de la théorie métrique des produits tensoriels topologiques, Boletim da Sociedade Matemática de São Paulo 8 (1953), 1-79. MR0094682 (20:1194)

[8] J. Lindenstrauss and A. Pełczyński, Absolutely summing operators in $\mathcal{L}_{p}$ spaces and their applications. Studia Math. 29 (1968), 275-326. MR0231188 (37:6743)

[9] M. C. Matos, Absolutely summing holomorphic mappings, An. Acad. Bras. Ci. 68 (1996), 1-13. MR1752625(2001c:46086)

[10] M. C. Matos, Nonlinear absolutely summing mappings, Math. Nachr. 258 (2003), 71-89. MR2000045 (2004g:47086)

[11] M. C. Matos, Fully absolutely summing mappings and Hilbert Schmidt operators, Collect. Math. 54 (2003), 111-136. MR1995136 (2004e:46052)

[12] D. Pérez-García, Operadores multilineales absolutamente sumantes, Doctoral Thesis, Universidad Complutense de Madrid, 2003.

[13] D. Pérez-García, The inclusion theorem for multiple summing operators, Studia Math. 165 (2004), 275-290. MR2110152 (2005i:47107)

[14] A. Pietsch, Absolute $p$-summierende Abbildungen in normierten Räumen, Studia Math. 28 (1967), 333-353. MR0216328 (35:7162)

[15] A. Pietsch, Ideals of multilinear functionals, in: Proceedings of the Second International Conference on Operator Algebras, Ideals and Their Applications in Theoretical Physics, TeubnerTexte, Leipzig, 1983, 185-199. MR.763541

Institute of Mathematics, University of Potsdam, 14469, Potsdam, Germany

E-mail address: junek@rz.uni-potsdam.de

IMECC-UNiCAMP, Caixa Postal 6065, Campinas, SP, Brazil

E-mail address: matos@ime.unicamp.br

Departamento de Matemática, UfPB, J. Pessoa, 58051-900, PB, Brazil

E-mail address: dmpellegrino@gmail.com 